\title{
Abdominal wall endometriosis versus desmoid tumor - a challenging differential diagnosis
}

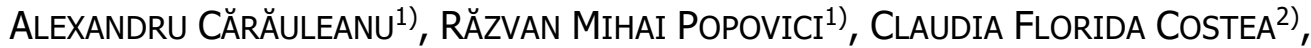

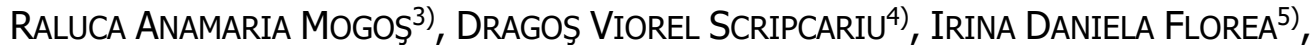

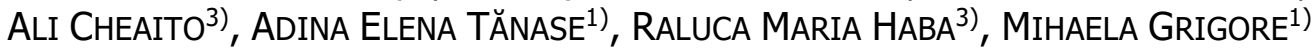 \\ 1)Department of Obstetrics and Gynecology, Faculty of Medicine, Grigore T. Popa University of Medicine and Pharmacy, Iaşi, \\ Romania \\ 2) Department of Ophthalmology, Faculty of Medicine, Grigore T. Popa University of Medicine and Pharmacy, Iaşi, Romania \\ ${ }^{3)}$ Cuza Vodă Hospital of Obstetrics and Gynecology, Iaşi, Romania \\ 4) Department of General Surgery, Faculty of Medicine, Grigore T. Popa University of Medicine and Pharmacy, Iaşi, Romania \\ ${ }^{5)}$ Department of Immunology, Faculty of Medicine, Grigore T. Popa University of Medicine and Pharmacy, Iaşi, Romania
}

\begin{abstract}
Aim: Abdominal wall endometriosis (AWE) in young women, with previous gynecological abdominal surgery, is the first condition considered by many practitioners when a tumor in the region of the scar appears. AWE seems to be caused by an iatrogenic transfer of endometrial cells at the level of the scar. The onset of the disease may be late in many cases. Despite the fact that the disease could be totally asymptomatic, there are certain risk factors that can be identified during the anamnesis, such as: heredity, menarche at the age of $>14$ years, menstrual cycle <27 days, delayed menopause, excessive alcohol and caffeine consumption. Suggestive signs include cyclic or continuous abdominal pain caused by a palpable abdominal wall mass with a maximum tenderness in the region of the surgical scar. The differential diagnosis is complex and rare entities like desmoid tumors (DTs) must be taken into consideration. Desmoid tumor, or the socalled aggressive fibromatosis (AF), is a rare fibroblastic proliferation. This tumor can develop in any muscular aponeurotic structure of the body and is considered benign but with a high recurrence rate. DTs can cause local infiltration, subsequently producing certain levels of deformity and potential obstruction of vital structures and organs. The differential diagnosis is challenging in this situations, the imagery exams are useful, especially in detecting the precise location of the tumor. The histological examination of the tumor can state the final and precise diagnosis.
\end{abstract}

Keywords: endometriosis, abdominal wall, desmoid tumor, differential diagnosis.

\section{ㅁ Introduction}

Abdominal wall tumors may have various etiologies. In young women with previous abdominal surgery, the diagnosis is difficult and different key points must be considered. The abdominal wall endometriosis (AWE) and the desmoid tumor (DT) are two entities with several similitude which make the differential diagnosis challenging. AWE occurs, in many cases, after obstetrical procedures such as a Caesarean (C)-section, which allows the transplantation of the endometrial tissue to ectopic sites, such as the abdominal wall. In 1903, the first case of abdominal endometriosis was reported in the literature by Meyer [1]. Even though, since then, many cases have been described in the literature, AWE remains an underreported pathology, partly due to the scant attention received in the radiological literature. DTs are benign lesions with an infiltrating growth pattern. Abdominal surgery may represent a predisposing factor for its development and the clinical appearance is similar to AWE [2]. However, at this moment, there are still several questions, difficult to answer when we face the diagnosis of a DT. The most important is "what is the most promising primary treatment (surgery, radiotheraphy, systemic therapy) in order to improve the prognosis and what is the best follow-up of these patients?".
The initial evaluation of an abdominal wall tumor is generally based on clinical exam and the medical history of the patient. Ultrasound (US) assessment, even though is widely used, can be misleading in the hands of an unexperienced examiner. The purpose of this paper is to highlight the main differences between AWE and DTs, contributing, in this way, to proper pre-operative diagnosis, which leads undoubtedly, in the long run to a better surgical outcome.

\section{a Abdominal wall endometriosis}

The condition occurs in both teenagers and women of reproductive age (10-15\%), but the etiology of the disease has not been elucidated yet. This disease is defined by the finding of endometrial tissue outside the uterine lining, in ectopic sites [1]. It frequently occurs in pelvis, the most common extrapelvic location of endometriosis is the abdominal wall (AWE) [2].

AWE mostly occurs at the level of the surgery scars following invasive abdominal-pelvic surgery, for instance after a C-section $(0.03-1 \%)$ or abdominal hysterectomy $(1.08-2 \%)$ [3]. There are reported cases of AWE after amniocentesis, hypertonic saline solution abortion, and laparoscopy $[4,5]$. AWE in these cases seems to be caused by an iatrogenic transfer of endometrial cells at the level

This is an open-access article distributed under the terms of a Creative Commons Attribution-NonCommercial-ShareAlike 4.0 International Public License, which permits unrestricted use, adaptation, distribution and reproduction in any medium, non-commercially, provided the new creations are licensed under identical terms as the original work and the original work is properly cited. 
of the scar. Studies show that the $\mathrm{C}$-section is a major risk factor, increasing the risk by 27 times [5]. The onset of the disease, in many cases, is a late one [4]; Hensen et al. (2006) confirms this aspect by a study conducted on 1500 women with C-section, of which only $12(0.8 \%)$ patients presented AWE that occurred even after seven years from the surgery [3].

Riazi et al. (2015) divide the positive diagnosis into three primary stages: anamnesis, physical examination and identification of the risk factors [5]. Typically, pelvic pain, dyspareunia and dysmenorrhea are the most frequent clinical findings in endometriosis [4]. Depending to the location of the disease, there are many signs or symptoms encountered, but their correlation to the menstrual cycle is strongly suggestive for the disease [1].

Studies show that there are certain risk factors that can be identified during the anamnesis: hereditary factors, menarche at the age of $>14$ years, menstrual cycle $<27$ days, delayed menopause, excessive alcohol and caffeine consumption [1]. In some instances, endometriosis can be totally asymptomatic and the positive diagnosis can be omitted [3].

The positive diagnosis of AWE can be established by clinical and paraclinical exams. Suggestive signs are: cyclic or continuous abdominal pain, chronic tiredness, sometimes associated with a tender mass proximal to the surgical scar $[6,7]$.

Savelli et al. (2012) show that $91 \%$ of the 21 female patients presented continuous or cyclical pain at the clinical examination, and only $9 \%$ were asymptomatic. The pain at the abdominal palpation was present in all cases included in the study. More than half of the female patients had dysmenorrhea, 33\% dyspareunia and $6 \%$ infertility [8].

Another study, published by Bozkurt et al. (2014) on a group of 445 patients with previous $\mathrm{C}$-section, showed that the main clinical sign was a solid tumor mass at the level of the scar, palpable in $96 \%$ of cases [9].

The anamnesis and physical examination are not enough to establish a positive diagnosis. The clinical examination has a poor predictive value; many of cases with surgically confirmed parietal endometriosis had a normal clinical examination. The imaging exams add a great value to the clinical examination $[10,11]$.

US examination has a good accuracy in establishing the diagnosis of AWE. Overall, in AWE, the US exam has a specificity of $83 \%$ and a sensitivity of $89 \%$ [8]. Generally, the mass is solid or hypoechogenic with variable sizes, sometimes with irregular margins. The Doppler examination may detect a vascular network within the tumor $[8,10,11]$ (Figure 1). The vascular pattern can be heterogeneous, represented by either poorly vascularized tumors or masses with abundant central vascularization due to multiple vessels originating in different areas of the tumor [10]. Growth limitation of the tumor appears in the subcutaneous fat due to scanty vascularization of this area. If the tumor size is greater than $15 \mathrm{~mm}$, Doppler velocimetry can show intralesional vascularization [9]. Usually, in the absence of previous surgery, a Doppler finding of central vascularity in an abdominal wall tumor is highly determinative for a malignancy of the soft tissue [8]. The differential diagnosis should be carefully assessed, with emphasis on the full history and clinical exam.
US scanning technique requires adapting the image size allowing an examination depth of 3-5 cm. On a transverse or longitudinal section, it is important to identify the normal abdominal anatomy of the parietal wall, at a certain distance from the origin of the tumor. US images of normal anatomical layers are as follows: subcutaneous tissue - hyperechoic, muscle layer - hypoechoic surrounded by the aponeurosis, which is hyperechoic. The peritoneum, a thin hyperechoic line covering the intestine, is difficult to assess in many instances. As a useful hint, the peritoneal fat located below the muscular layer, may be of great use in the localization of the peritoneum. Exerting slight pressure and sliding the probe to the site of maximum pain induced will help to locate the AWE. The aspect of the AWE when examined in two-dimensional US is a heterogeneous hypoechoic mass with either small echogenic foci or large echogenic strands, depending on the proportion of fibrosis of the abdominal wall [8-10] (Figure 2).

Macroscopically, the abdominal wall has a pink aspect due to the endometriotic tissue and may present one or multiple bluish cystic masses. In some instances, micro-cysts with a chocolate-like appearance (ovarian endometrioma-like) can be clearly identified in the surrounding subcutaneous fat, which has a yellowish aspect. In cases with muscular involvement, the endometriotic mass can be easily distinguished due to its irregular appearance and hard consistency. Rarely, it can occur as multiple polypoid masses, closely resembling a neoplastic process (also named polypoid endometriosis or endometriotic polyposis) $[6,12,13]$.

Microscopically, the process consists of endometrial glands and stroma associated with dense fibrous tissue and signs of fresh and old hemorrhage [12, 14] (Figure 3). Sometimes, the stromal component of endometriosis can undergo smooth muscle metaplasia. From histological point of view, in AWE is described a cytogenetic chorion associated with a hyperplasia of smooth muscle fibers within the soft tissues, musculature of abdominal and pelvic wall, inflammatory cells, different levels of fibrosis and a granulomatous reaction against suture material (Figure 4). The endometriotic glands can sometimes show mucinous metaplasia, a feature that can be confused with well-differentiated mucinous adenocarcinoma [13].

The differential diagnosis includes neoplasms: sarcomas, DTs, lymphomas, metastases, and non-neoplastic causes, such as: granulomas, hematomas, abdominal soft tissue abscesses and ventral hernias [10, 14].

In cases presenting a soft tissue mass, for describing the precise structure of the lesion and its surrounding structures, magnetic resonance imaging (MRI) is considered the best method. It is preferred in younger patients, due to the absence of ionizing radiation. MRI has the advantage of diagnosing even very small lesions and at the same time, offers a better image of the interlining between muscles and abdominal subcutaneous tissues and provides information regarding the infiltration of abdominal and pelvic wall structures [15]. In both T1 and T2 MRI sequences, AWE appears as a hyperintense heterogeneous mass associated with a surgical scar. A very suggestive MRI aspect for parietal endometriosis is a well-delimited subcutaneous solid mass with the infiltration of the muscular 
aponeurosis. In some instances, when scar endometriosis is more isointense than muscular tissue on T1-weighted images, the diagnosis can be difficult to establish due to the lack of differentiation between tumor and muscular

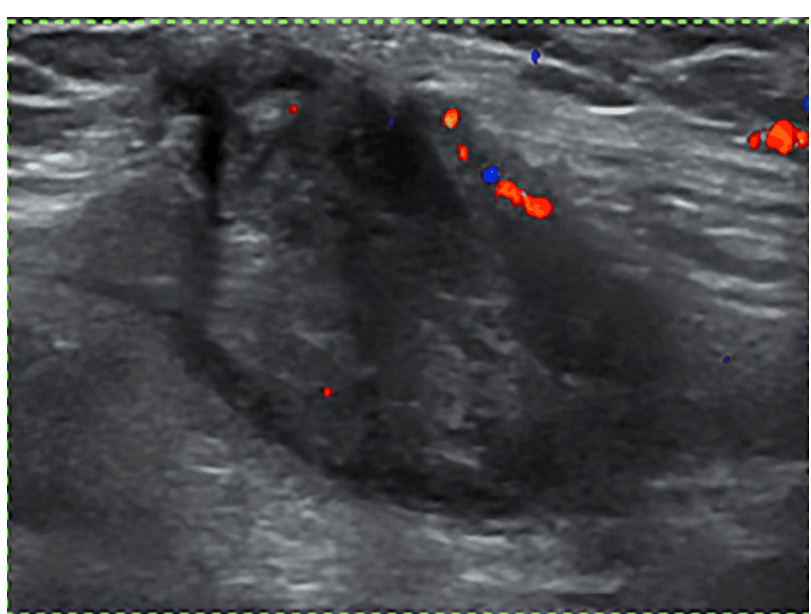

Figure 1 - Abdominal wall endometriosis. Color Doppler US image showing peripheral flow within the mass. US: Ultrasound.

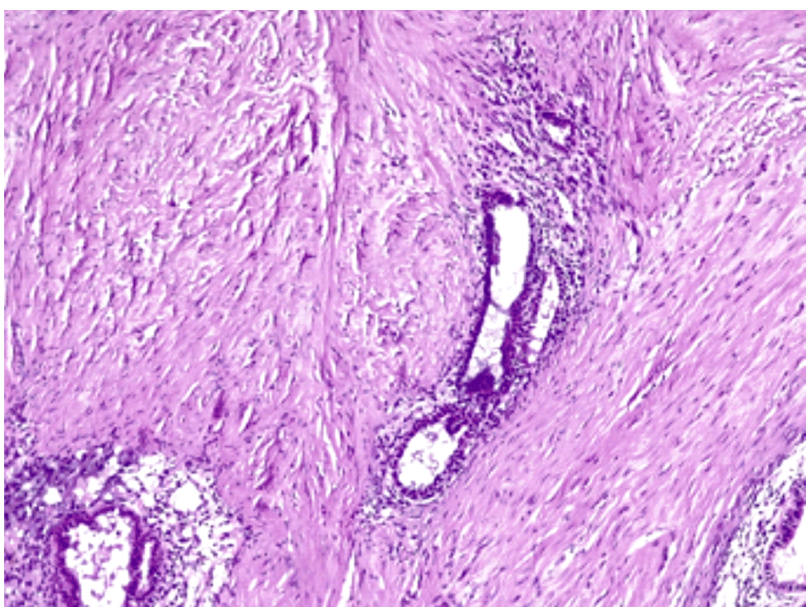

Figure 3 - Fibrous tissue containing endometrial glands surrounded by endometrial stroma, aspects that are consistent with the diagnosis of abdominal wall endometriosis. HE staining, $\times 40$. HE: Hematoxylin-Eosin.

In women with abdominal wall masses, US-guided fine-needle aspiration (FNA) provides, in the majority of cases, a precise diagnosis. FNA may be helpful in the differential diagnosis between benign and malignant conditions. Because endometriosis nodules are firm, in many cases, the procedure is easy to perform, and can confirm the diagnosis even in isolated cases [16]. Epithelial endometrial-like cells and hemosiderin-laden macrophages are pathognomonic findings in aspiration samples. The presence of fibrotic tissue in cases of old lesions of endometriosis or a limited amount of sample material may lead to an inconclusive result and additional histological biopsy may be considered [17]. The controversy regarding the possibility of generating new inseminations at the puncture sites led to the recommendation of including tissue [16]. In old scar endometriosis, lesions may have spiculated margins and low signal intensity on T2-weighted images, caused by both dominant fibrotic and hemosiderotic components $[15,16]$.

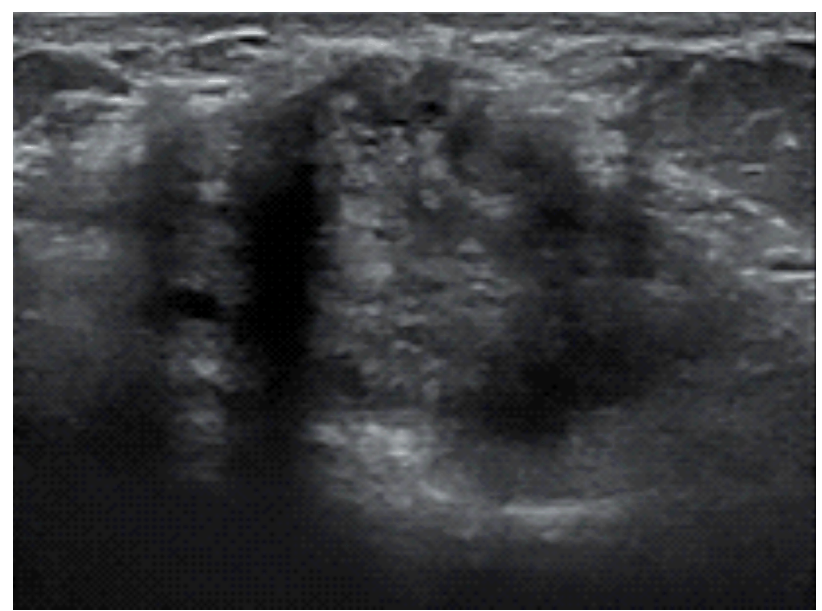

Figure 2 - Transverse sonogram of the abdominal wall showing an abdominal wall endometriosis nodule with hypoechoic content and blurred outer margins. The nodule is enclosed in the subcutaneous fatty tissue, above the muscular fascia, along the scar of a previous Caesarean section.

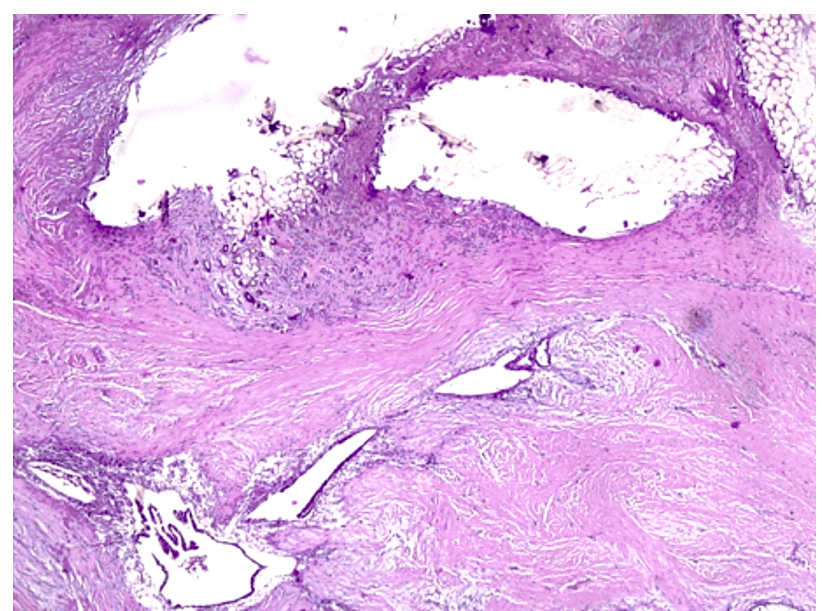

Figure 4 - Fibrous tissue containing, in the upper part, aspects of granulomatous reaction against suture threads (due to a previous caesarean section) and in the lower part, endometrial glands surrounded by endometrial stroma, aspects consistent with abdominal wall endometriosis. HE staining, $\times 40$.

the site of aspiration in the field of operative resection [18].

Laparoscopy with biopsy showing benign fibrous tissue with multiple endometrial glands remains the "gold standard" for the diagnosis [16]. Nevertheless, there are new studies emphasizing the presence of certain biomarkers that can be used for a positive diagnosis: increased levels of cancer antigen-125 (CA-125), growth factors [transforming growth factor-beta 1 (TGF- $\beta 1)$ ], intercellular adhesion molecule-1 (ICAM-1), cluster of differentiation (CD) 9, CD34 [1].

The risk of malignization in AWE is low, about $1 \%$ of cases [14]. The malignant transformation of endometriosis is related to certain factors, such as: advanced age of the patient, postmenopausal status and tumor diameter more 
than $9 \mathrm{~cm}[1,14]$. The time frame of a possible transformation varies from a few months to 18 years after surgery. The most common histological type is clear cell carcinoma with a 20 -month survival rate of $57 \%$ [1]. In the literature, there have been described some rare types, such as: carcinosarcoma, cystadenocarcinoma, and serous papillary carcinoma. Wide excision of AWE with clear margins represents the main prevention method $[4,5$, $14,16]$.

\section{口 Desmoid tumors}

Desmoid tumor, or the so-called aggressive fibromatosis (AF), is a rare fibroblastic proliferation [18]. MacFarlane first described it in 1832. This tumor can develop in any muscular aponeurotic structure of the body [19] and is considered benign for several reasons: it is histologically characterized by normal mitosis and never metastasizes. The recurrence rate is high, even after its surgical resection [19]. There are several known risk factors involved in the occurrence of the disease: previous surgeries, pregnancy, estrogen treatment, oral contraceptives and familial polyposis $[19,20]$.

The increasing risk of distant recurrence seems to have predictive factors, such as: age less than 37 years, female gender, poor general condition at admission, tumor size $>7 \mathrm{~cm}$, extra-abdominal location [21, 22].

Studies have shown that there are several types of AF: $(i)$ sporadic (rare): frequently, the location is at the level of the abdominal wall, (ii) associated to familial adenomatous polyposis (FAP) in $10-25 \%$ of cases and (iii) hereditary (extremely rare) [23].

DTs normally do not express estrogen receptors but Tamoxifen treatment is effective in a considerable number of cases [24]. The disease occurs more frequently in female patients during their reproduction period, especially during pregnancy or in women with previous C-section [23]. Couto Netto et al. present a study of 32 patients diagnosed with DT; $90 \%$ of them were female, $62.5 \%$ with previous pregnancies and $19 \%$ with surgical of the abdominal wall [25].

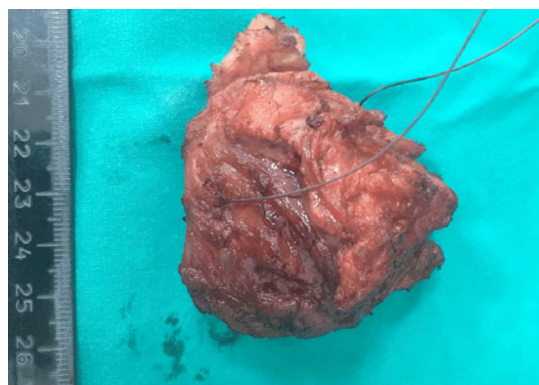

Figure 5 - Macroscopic view of an excised abdominal wall mass, subsequently confirmed as desmoid tumor.

US differential diagnosis of a mass in the abdominal wall is difficult to assess, and a wide spectrum of disorders should be taken in consideration. This includes tumors of different origins like desmoid, lymphomas or metastatic tumors. Also, non-neoplastic causes should be considered, including suture granuloma, ventral hernia, retained surgical material, parietal abscess or hematoma. At US exam,
Leon et al. (2015) present the case of a 24-year-old woman, pregnant in the $14^{\text {th }}$ week of gestation, presenting a palpable abdominal mass at the clinical examination. US revealed a tumor of $11.7 \times 12.3 \times 17 \mathrm{~cm}$, located in the lower left abdominal quadrant. In the first instance, a uterine fibroid was suspected. After five weeks, US detected an increase in the tumor size $(16.5 \times 11.8 \times 18.5 \mathrm{~cm})$. MRI confirmed the location of the tumor mass at the level of the left rectus abdominis muscle. Considering the patient's risk factors and the location of the tumor, a DTs was suspected, and later confirmed by the histological exam [26].

Another case was published in the article "Abdominal wall desmoid tumors: a case report", in which Ma et al. (2013) presented the case of a 17-year-old patient with a firm abdominal mass adherent to the abdominal wall, painful at palpation. Computed tomography (CT) examination revealed the intramuscular position of the tumor. It has to be specified that the patient was not pregnant that time and did not have any previous surgical intervention $\mathrm{s}$ and the tumoral markers were negative. The biopsy confirmed the diagnosis of DTs [22].

There is a high potential for DTs to cause local infiltration, subsequently producing certain levels of deformity and potential obstruction of vital structures and organs (Figure 5).

The main treatment is surgery with wide excision and negative margins. Subsequent radiotherapy may decrease the local recurrence rate [27].

The positive diagnosis of DT is not always easy, it and can be often confused with parietal endometriosis due to the fact that the clinical signs are similar and the imaging exams are not specific. US can guide the diagnosis by locating the tumor: an intramuscular or aponeurotic site indicates mostly a DT, whilst a subcutaneous topography may be suggestive for an endometrioma. The main US features of DTs are: oval shape, imprecisely delimited margins, different hypo- and hyperechogenic areas [28] (Figure 6a). Doppler pattern suggests the presence of blood vessels within the tumor [28] (Figure 6b). a

Figure 6 - ( $(a$ and b) Desmoid tumor. US image - transverse plane of the abdominal wall: hypoechoic nodule with regular contour. US: Ultrasound.

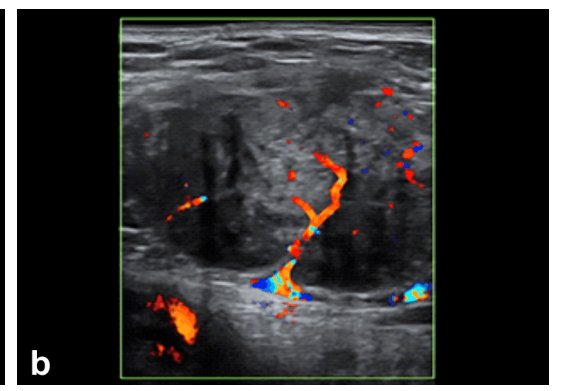

acute hematomas are usually hyperechogenic, whereas resolving hematomas or seromas are hypoechoic or anechoic. An air-fluid level is often observed in abscesses. The clinical history plays an important role, knowing that both hematomas and abscesses manifest in the setting of a recent surgery. Lymphoceles are usually anechoic and septated. A granuloma presents an irregular shape and 
inhomogeneous, hyper- and hypoechoic structure, a biopsy being required in most cases [16].

US appearance of metastases consists in poorly defined hypoechoic masses, with increased vascularity. In lymphomas, US exam provides nonspecific and variable aspects, from a large mass with nodal structures to small nodules $(<1 \mathrm{~cm})$ or disseminated myositis and panniculitis $[4,16]$.

Difficulties may occur in the differential diagnosis of the DTs. Typically, these tumors do not present cyclical pain, but imagery and clinical exam should be carefully taken in consideration, having in mind that the US pattern of a DT may be, in many cases, similar to those encountered in scar endometriosis. At US examination, the DT, usually, has a hypoechoic aspect, is relative small with well-defined limits [16].

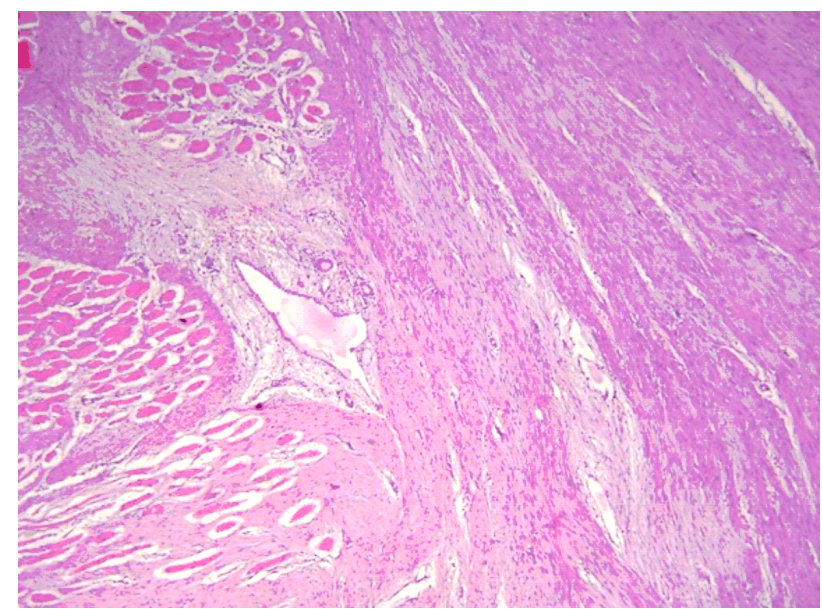

Figure 7 - Desmoid tumor. Spindle cells of desmoid tumor, with bland appearance grow diffusely and dissociate skeletal muscle fibers; the tumor associates angulated medium size blood vessels and collagenic stroma. HE staining, $\times 40$.

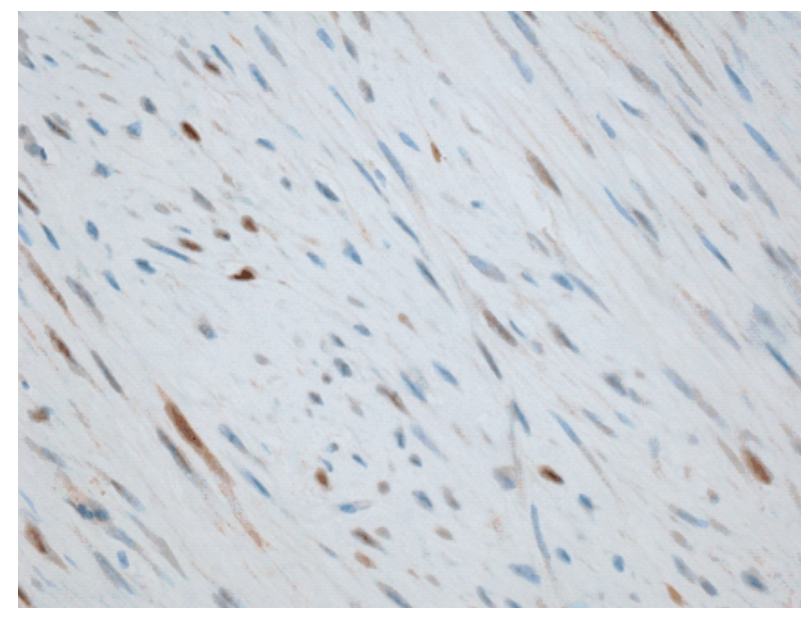

Figure 9 - Desmoid tumor cells with nuclear positivity for cyclin D1. Anti-cyclin D1 antibody immunostaining, $\times 400$.

\section{Conclusions}

An accurate presurgical diagnosis of an abdominal wall tumor, especially in young women with known history of gynecological abdominal surgery, is difficult to obtain. The endometriosis of the abdominal wall is always taken
The biopsy allows the certainty of the diagnosis [23]. DTs are, grossly, usually large lesions, poorly defined, whitish and firm. Microscopically, in the conventional form, they consist of a proliferation of long fascicles containing uniform spindle cells, which are dissociated by a usually abundant amount of collagenic stroma (Figure 7). The cells lack or have low cytological atipia and have variable mitotic rate (usually low but can be focally increased) (Figure 8). The unconventional forms of DTs include the hypo-/hypercellular forms, myxoid pattern, keloidal, nodular fasciitis-like and staghorn-vessel pattern [29]. DTs show variable positivity for alpha-smooth muscle actin ( $\alpha$-SMA) and only rarely and focally for desmin [30]. They are in more than $70 \%$ of cases positive for $\beta$-catenin and in around $70 \%$ of cases positive for calretinin and cyclin D1 [31] (Figure 9).

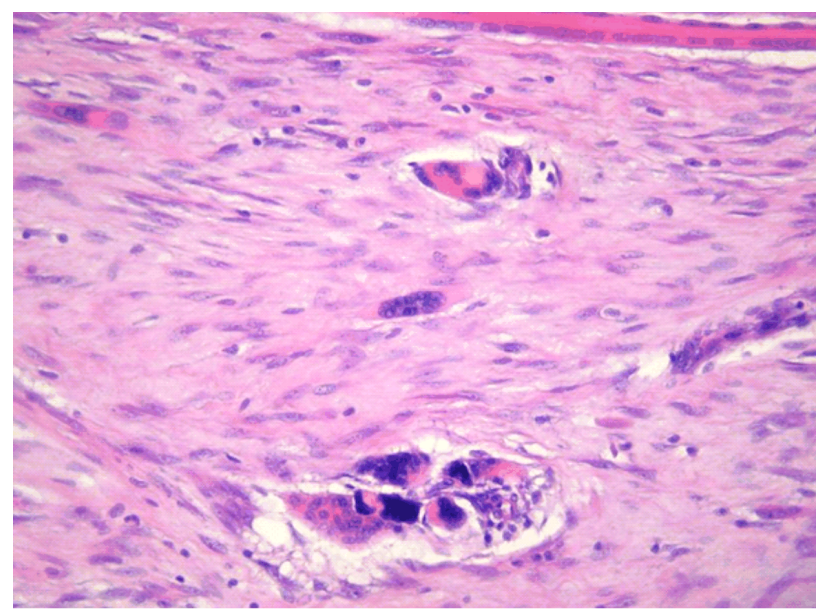

Figure 8 - Desmoid tumor. Atrophic muscle fibers enclosed by the desmoid tumor spindle cells showing uniform and bland appearance. HE staining, $\times 400$.

in consideration in these cases, but imaging exams showing an intramuscular location of the tumor and a known history of familial polyposis could suggest the presence of a DT. Surgery is the main treatment and the histological exam is mandatory in shaping the precise diagnosis.

\section{Conflict of interests}

The authors declare that they have no conflict of interest.

\section{References}

[1] Parasar P, Ozcan P, Terry KL. Endometriosis: epidemiology, diagnosis and clinical management. Curr Obstet Gynecol Rep, 2017, 6(1):34-41. https://doi.org/10.1007/s13669-017-0187-1 PMID: 29276652 PMCID: PMC5737931

[2] Anand M, Deshmukh SD. Massive abdominal wall endometriosis masquerading as desmoid tumour. J Cutan Aesthet Surg, 2011, 4(2):141-143. https://doi.org/10.4103/0974-2077. 85043 PMID: 21976909 PMCID: PMC3183722

[3] Hensen JHJ, Van Breda Vriesman AC, Puylaert JBCM. Abdominal wall endometriosis: clinical presentation and imaging features with emphasis on sonography. AJR Am J Roentgenol, 2006, 186(3):616-620. https://doi.org/10.2214/ AJR.04.1619 PMID: 16498086

[4] Paşalega M, Mirea C, Vîlcea ID, Vasile I, Pleşea IE, Calotă F, Meşină C, Pârvănescu H, Baluta M. Parietal abdominal endometriosis following Cesarean section. Rom J Morphol Embryol, 2011, 52(1 Suppl):503-508. PMID: 21424102 
[5] Riazi H, Tehranian N, Ziaei S, Mohammadi E, Hajizadeh E, Montazeri A. Clinical diagnosis of pelvic endometriosis: a scoping review. BMC Womens Health, 2015, 15:39. https:// doi.org/10.1186/s12905-015-0196-z PMID: 25952159 PMCID: PMC4450847

[6] Alimi Y, Iwanaga J, Loukas M, Tubbs RS. The clinical anatomy of endometriosis: a review. Cureus, 2018, 10(9):e3361. https:// doi.org/10.7759/cureus.3361 PMID: 30510871 PMCID: PMC 6257623

[7] Troyer MR. Differential diagnosis of endometriosis in a young adult woman with nonspecific low back pain. Phys Ther, 2007 87(6):801-810. https://doi.org/10.2522/ptj.20060141 PMID: 17442838

[8] Savelli L, Manuzzi L, Di Donato N, Salfi N, Trivella G, Ceccaroni M, Seracchioli R. Endometriosis of the abdominal wall: ultrasonographic and Doppler characteristics. Ultrasound Obstet Gynecol, 2012, 39(3):336-340. https://doi.org/10.10 02/uog.10052 PMID: 21793086

[9] Bozkurt M, Çil AS, Bozkurt DK. Intramuscular abdominal wall endometriosis treated by ultrasound-guided ethanol injection. Clin Med Res, 2014, 12(3-4):160-165. https://doi.org/10.31 21/cmr.2013.1183 PMID: 24667221 PMCID: PMC4317157

[10] Brosens I, Puttemans P, Campo R, Gordts S, Brosens J. Non-invasive methods of diagnosis of endometriosis. Curr Opin Obstet Gynecol, 2003, 15(6):519-522. https://doi.org/ 10.1097/00001703-200312000-00011 PMID: 14624220

[11] Gourgiotis S, Veloudis G, Pallas N, Lagos P, Salemis NS, Villias C. Abdominal wall endometriosis: report of two cases. Rom J Morphol Embryol, 2008, 49(4):553-555. PMID: 19050806

[12] Krentel H, Tchartchian G, De Wilde RL. Desmoid tumor of the anterior abdominal wall in female patients: comparison with endometriosis. Case Rep Med, 2012, 2012:725498. https:// doi.org/10.1155/2012/725498 PMID: 22778752 PMCID: PMC 3385014

[13] Istrate-Ofiţeru AM, Pirici D, Niculescu M, Berceanu C Berceanu S, Voicu NL, Piringă GD, Roşu GC, lovan L, Căpitănescu RG, Diţescu D, Sava A, Mogoantă L, Neacşu A. Clinical, morphological and immunohistochemical survey in different types of endometriosis. Rom J Morphol Embryol, 2018, 59(4):1133-1153. PMID: 30845295

[14] Mostoufizadeh M, Scully RE. Malignant tumors arising in endometriosis. Clin Obstet Gynecol, 1980, 23(3):951-963. PMID: 7418292

[15] Saliba C, Jaafoury H, El Hajj M, Nicolas G, Haidar Ahmad H. Abdominal wall endometriosis: a case report. Cureus, 2019, 11(2):e4061. https://doi.org/10.7759/cureus.4061 PMID: 31016088 PMCID: PMC6464279

[16] Grigore M, Socolov D, Pavaleanu I, Scripcariu I, Grigore AM, Micu R. Abdominal wall endometriosis: an update in clinical, imagistic features, and management options. Med Ultrason, 2017, 19(4):430-437. https://doi.org/10.11152/mu-1248 PMID 29197920

[17] Clement PB. Pathology of endometriosis. Pathol Annu, 1990 25(Pt 1):245-295. PMID: 2404246

[18] Ravi V, Patel SR, Raut CP, DeLaney TF. Desmoid tumors: epidemiology, risk factors, molecular pathogenesis, clinical presentation, diagnosis, and local therapy. UpToDate, literature review current through: Mar 2020, available from: https:// www.uptodate.com/contents/desmoid-tumors-epidemiologyrisk-factors-molecular-pathogenesis-clinical-presentationdiagnosis-and-local-therapy.
[19] Wirth L, Klein A, Baur-Melnyk A, Knösel T, Lindner LH, Roeder F, Jansson V, Dürr HR. Desmoid tumours of the extremity and trunk. A retrospective study of 44 patients. BMC Musculoskelet Disord, 2018, 19(1):2. https://doi.org/10.1186/s12891-017-19 24-3 PMID: 29304783 PMCID: PMC5756424

[20] Escobar C, Munker R, Thomas JO, Li BD, Burton GV. Update on desmoid tumors. Ann Oncol, 2012, 23(3):562-569. https:// doi.org/10.1093/annonc/mdr386 PMID: 21859899

[21] Liu X, Zong S, Cui Y, Yue Y. Misdiagnosis of aggressive fibromatosis of the abdominal wall: a case report and literature review. Medicine (Baltimore), 2018, 97(10):e9925. https:// doi.org/10.1097/MD.0000000000009925 PMID: 29517699 PMCID: PMC5882433

[22] Ma JH, Ma ZH, Dong XF, Yin H, Zhao YF. Abdominal wall desmoid tumors: a case report. Oncol Lett, 2013, 5(6):19761978. https://doi.org/10.3892/ol.2013.1297 PMID: 23833679 PMCID: PMC3700980

[23] Shinagare AB, Ramaiya NH, Jagannathan JP, Krajewski KM, Giardino AA, Butrynski JE, Raut CP. A to Z of desmoid tumors. AJR Am J Roentgenol, 2011, 197(6):W1008-W1014. https:// doi.org/10.2214/AJR.11.6657 PMID: 22109314

[24] Libertini M, Mitra I, van der Graaf WTA, Miah AB, Judson I, Jones RL, Thomas K, Moskovic E, Szucs Z, Benson C, Messiou C. Aggressive fibromatosis response to tamoxifen: lack of correlation between MRI and symptomatic response. Clin Sarcoma Res, 2018, 8:13. https://doi.org/10.1186/s13 569-018-0100-3 PMID: 29785261 PMCID: PMC5950191

[25] Couto Netto SD, Teixeira F, Menegozzo CAM, Leão-Filho HM, Albertini A, Ferreira FO, Akaishi EH, Utiyama EM. Sporadic abdominal wall desmoid type fibromatosis: treatment paradigm after thirty two years. BMC Surg, 2018, 18(1):37. https://doi. org/10.1186/s12893-018-0367-6 PMID: 29879959 PMCID: PMC5992671

[26] Leon MG, Moussa HN, Movahedian M, Viteri OA, Longo M, Sibai BM. A rapidly growing abdominal mass: desmoid tumor in pregnancy. AJP Rep, 2015, 5(1):e14-e17. https://doi.org/ 10.1055/s-0034-1396028 PMID: 26199790 PMCID: PMC 4502627

[27] Wanjeri JK, Opeya CJ. A massive abdominal wall desmoid tumor occurring in a laparotomy scar: a case report. World J Surg Oncol, 2011, 9:35. https://doi.org/10.1186/1477-78199-35 PMID: 21426541 PMCID: PMC3070676

[28] Braschi-Amirfarzan M, Keraliya AR, Krajewski KM, Tirumani SH, Shinagare AB, Hornick JL, Baldini EH, George S, Ramaiya NH, Jagannathan JP. Role of imaging in management of desmoidtype fibromatosis: a primer for radiologists. Radiographics, 2016, 36(3):767-782. https://doi.org/10.1148/rg.2016150153 PMID: 27163593

[29] Zreik RT, Fritchie KJ. Morphologic spectrum of desmoid-type fibromatosis. Am J Clin Pathol, 2016, 145(3):332-340. https:// doi.org/10.1093/ajcp/aqv094 PMID: 27124915

[30] Heim-Hall J, Yohe SL. Application of immunohistochemistry to soft tissue neoplasms. Arch Pathol Lab Med, 2008, 132(3): 476-489. https://doi.org/10.1043/1543-2165(2008)132[476:A OITST]2.0.CO;2 PMID: 18318588

[31] Barak S, Wang Z, Miettinen M. Immunoreactivity for calretinin and keratins in desmoid fibromatosis and other myofibroblastic tumors: a diagnostic pitfall. Am J Surg Pathol, 2012, 36(9): 1404-1409. https://doi.org/10.1097/PAS.0b013e3182556def PMID: 22531174

\section{Corresponding authors}

Claudia Florida Costea, Associate Professor, MD, PhD, Department of Ophthalmology, Faculty of Medicine, Grigore T. Popa University of Medicine and Pharmacy, 16 University Street, 700115 laşi, Romania; Phone +40744-972 648, e-mail: costea10@yahoo.com

Răzvan Mihai Popovici, Assistant, MD, PhD, Department of Obstetrics and Gynecology, Faculty of Medicine, Grigore T. Popa University of Medicine and Pharmacy, 16 University Street, 700115 laşi, Romania; Phone +40744-596 918, e-mail: razpopovici@yahoo.com 